\title{
Initial droplet conditions in numerical spray painting by electrostatic rotary bell sprayers
}

\author{
A framework for optimization of injection model coefficients
}

\author{
Nico Guettler, Philipp Knee, Qiaoyan Ye, Oliver Tiedje
}

(C) The Author(s) 2020

\begin{abstract}
In computational fluid dynamics, the modeling of paint application processes by electrostatic rotary bell sprayer is mostly performed using an EulerLagrange approach. The initial conditions of the discrete phase-position, velocity, size, and chargehave an essential influence on the resulting film thickness distribution and the total charge transferred to the object. Typically, so-called injection models are used to specify these initial conditions, whereby the determination of the injection model coefficients is crucial. In this paper, a framework is proposed that combines experimental input data, an injection model, and a metamodel-based optimization. The painting tests for the generation of input and validation data were carried out in a technical center in the industrial standard. The simulations were performed using ANSYSFluent. Initial droplet conditions could efficiently be determined via the framework so that the painting-specific objectives were achieved with reasonable accuracy. In addition to the framework, a turbulence study of the strongly swirled shaping air of this atomizer was carried out, whereby a substantial underestimation of the axial air velocity was found in the turbulence models being investigated. The initial droplet conditions were also used in this study to draw conclusions about the accuracy of the airflow simulation. The proposed framework can be adapted to other solvers and efficiently finds injection model coefficients for other paint applicators.
\end{abstract}

This paper was presented at the 15 th Coatings Science International Conference on June 24-29, 2019, in Noordwijk, the Netherlands.

N. Guettler $(\varangle)$, P. Knee, Q. Ye, O. Tiedje

Fraunhofer Institute for Manufacturing Engineering and

Automation IPA, Nobelstrasse 12, 70569 Stuttgart,

Germany

e-mail: nico.guettler@ipa.fraunhofer.de
Keywords Spray painting simulation, Rotary bell atomizer, Electrostatic painting simulation, Metamodel

\section{Introduction}

Numerical simulations of paint application processes are used for virtual operating trials to perform paintability studies in early design phases, to optimize process parameters, and to reduce the number of painting prototypes. To reduce the computational effort and increase the accuracy of painting simulations, both numerical and painting-specific models and methods must be continuously enhanced. Particularly in spray simulations, the Euler-Lagrange approach requires injection models to determine the initial conditions-position, velocity, size, and charge of the particles. In this study, an electrostatic rotary bell sprayer (ERBS) was used, which is being used in the automotive industry to paint car bodies and plastic parts like bumpers. When using ERBS, paint is stationary added inside the bell-cup through a small orifice on the rotation axis. Due to the rotational speed of the bellcup, the paint is distributed radially on the inner surface by centrifugal forces. At the bell-edge, the paint is atomized because of the high relative velocity between the liquid and gaseous phases. The shaping air causes a change in the direction of the droplet movement by a strong axial component toward the object to be painted. The electrostatic field between the charged bell-cup, shaping air ring, and the grounded substrate increases the transfer efficiency of the paint by applying an additional force to the charged particles (see Fig. 1).

In general, rotary atomizers have been investigated in various fields of application, such as spray drying, spray painting, or granulate production, where different types of disks and bell-cups both with and without shaping air were being used. Investigations on the film 


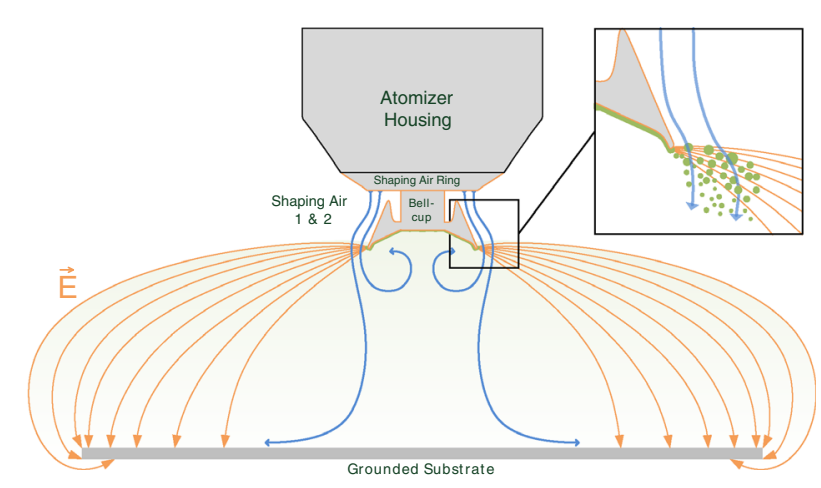

Fig. 1: Functional principle of paint application using electrostatic rotary bell sprayer

thickness of a liquid within a rotating conical body were performed by references (1), (2), and (3) who derived a formula to calculate the film flow velocity on the inner bell-cup surface. Reference (2) also showed that the disintegration process on rotating cups could be divided into three modes: direct drop formation, ligament formation, and film formation. The transitions between these modes were also experimentally investigated by references (4) and (5) on a rotating disk as well as by references (6) and (7) on a rotating bellcup. However, there are very few numerical works that have investigated the primary breakup of paint liquids by ERBS in a full computational framework, for example, references (8) and (9). In their work, paint film distribution on the bell cup and the primary breakup of liquid quite close to the bell edge were experimentally and numerically investigated, which delivers some useful information for the particle trajectory calculation using the Euler-Lagrange method. Due to the complex properties of paint materials (non-Newtonian, shear-thinning, and thixotropic fluid), the simulation of the primary atomization is only possible with very high computational effort and wellknown material properties, which is why the EulerLagrange approach is preferred for paint simulations in the industry and industrial applied science.

Detailed spray simulations have already been performed and evaluated by reference (10). The authors also described that the spray behavior strongly depends on the initial conditions of the discrete phase and must be calibrated to experimental data. However, the dependencies of the initial droplet conditions were not directly shown, and no framework was derived to determine the injection parameters. Recently published work by reference (11) presents an injection model for ERBS in which the injection model consists of multiple rings with different amounts of injection points, radii, angles, and mass flow rates. It was shown that simulation results could be improved with steeper injection angles to the object, respectively, higher axial velocity. However, in this model, no dependence on the charge of the droplets was shown. Reference (12) also presents a parameter study on an injection model by systematically investigating injection positions and velocities. The author concludes that the axial injection position, as well as the radial and tangential injection velocity, has no significant influence on the spray pattern, whereas the radial position and the axial velocity clearly affect the simulation result.

Numerical investigations on the effects of electrostatically assisted paint application have already been carried out by references (10), (11), (12), (13), and (14), using different models to distribute the charge on the droplets as a function of droplet diameter. A study on the influence of different models of particle charge distribution on the resulting film thickness distribution was performed by reference (13). The authors concluded that the transfer efficiency increases with increasing charge on the droplet surface, but no proposal to the use of a specific model was derived.

This paper proposes an injection model and a framework to calibrate the injection model coefficients by a metamodel-based optimization in combination with experimental painting-specific data. Afterwards a simulation with a complex workpiece including moving atomizers along a robot path will be simulated with the optimized injection parameters.

The paper is divided into seven main sections. First, an overview of the entire framework is presented in "Structure and objective of the framework" section. "Experimental materials and methods" and "Numerical methods" sections describe the experimental, numerical materials and methods used in this framework. The results of a turbulence study of the shaping air flow are discussed in "Simulation of the airflow field" section. An injection model for this ERBS is proposed in "Modeling initial droplet conditions" section. The effective determination of the injection model coefficients is then described and discussed in "Metamodel-based optimization of injection model coefficients" section. The results and procedure are finally discussed, and a conclusion with an outlook on further research work is given.

\section{Structure and objective of the framework}

The considerable variation of paint properties in combination with system-specific or customer-specific ERBS equipment and process parameters makes it difficult to define a generic model for the initial droplet conditions in painting simulations. The framework is presented using an ERBS but can also be used efficiently for other atomizers, such as high volume low pressure or airless atomizers. Figure 2 illustrates the workflow of the proposed framework in form of a high-level sketch. The majority of the workflow aims to create metamodels. The metamodels are used in the subsequent step of multiobjective optimization to make the very high number of function evaluations more efficient. First, a feasible range for the injection model coefficients must be defined. Using a LatinHypercube experiment design, a set of sampling points is generated within the feasible space. Based on this, a set of simulations will be carried out and evaluated 
with regard to the painting-specific objectives. Correlation coefficients are used to identify the principal components of the model.

The framework assumes that experimental data on the paint material (e.g., density and solids content), as well as process-related data (e.g., spray pattern, discharge current, and particle size distribution), are available. Often a large part of this data is already available or can be generated relatively inexpensively in paint shops.

\section{Experimental materials and methods}

This section describes the equipment and process settings used as well as the experimental methods in more detail.

\section{Spray booth, atomizer, and paint material}

The experimental investigations were carried out in an environmentally controlled spray booth with an

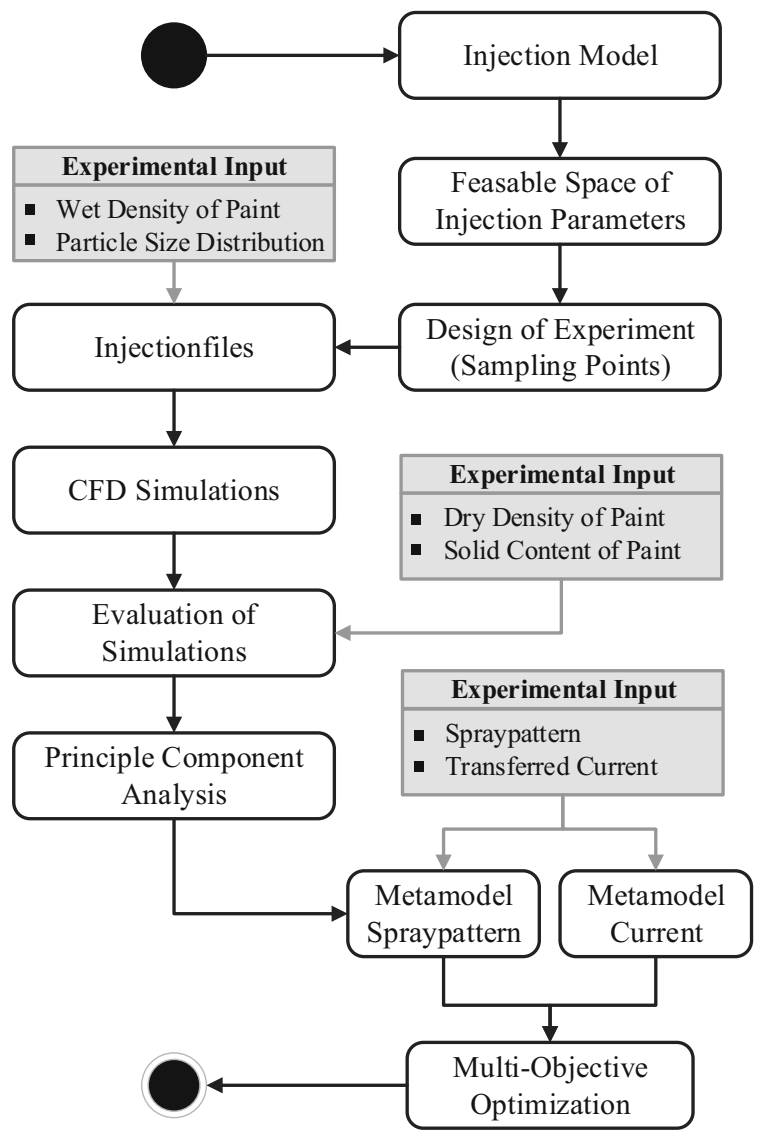

Fig. 2: High-level sketch of the framework for the determination of the optimized initial droplet conditions. Gray boxes indicate experimental input data are needed in this step ambient temperature of $23^{\circ} \mathrm{C}$, a relative humidity of $60 \%$, and a vertical booth airflow of $0.3 \mathrm{~ms}^{-1}$. The ERBS picoBell HiBlow III of the company EISENMANN-LACTEC with a $50 \mathrm{~mm}$ bell-cup (no serration) and a direct charging mechanism was chosen and mounted on a 6-axis ABB robot. The shaping air ring has 40 outer and 32 inner nozzles, which are referred to in the following as $\mathrm{SA}_{\text {out }}$ and $\mathrm{SA}_{i n}$. The outer and inner nozzles are oriented tangentially to the axis of rotation but have different axial angles (see Fig. 3). The different orientation of $\mathrm{SA}_{\text {out }}$ and $\mathrm{SA}_{\text {in }}$ enable a purposeful forming of the airflow to paint complex geometries, e.g., bumpers, efficiently. An application setting was chosen, which is used in industrial paint shops. In addition to the shaping air, the process settings are determined by the paint flow rate $\mathrm{PF}$, the revolutions per minute of the bell-cup $\mathrm{n}_{\text {bell }}$, the high voltage HV applied to the bellcup and the shaping air ring, and the painting distance $\mathrm{d}$ (also referred as the distance to bell-edge). The paint application process setting is shown in Table 1. A twocomponent solvent-borne industrial coating with a wet density of $1109.5 \mathrm{~kg} \mathrm{~m}^{-3}$, a dry density of $1293.8 \mathrm{~kg} \mathrm{~m}^{-3}$, and a nonvolatile content of $67.9 \%$ was used as painting material.

\section{Determination of shaping air velocity}

The airflow velocity of the shaping air was determined using a DANTEC 2-component fiber Laser-Doppler Anemometry (LDA) system. As tracer particles, glycerine mist was injected into the intake flow above the

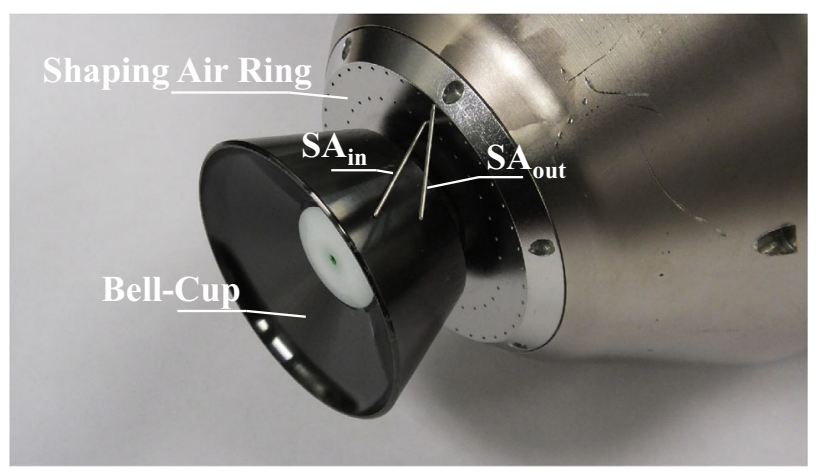

Fig. 3: Simulation-relevant components of the atomizer. Needles were drilled into the shaping air nozzles to visualize their orientation

Table 1: Paint application process setting

\begin{tabular}{|c|c|c|c|c|}
\hline $\begin{array}{l}\mathrm{PF} \\
\mathrm{ml} \min ^{-1}\end{array}$ & $\begin{array}{c}\mathrm{SA}_{\text {out }} \\
\mathrm{I}_{S} \mathrm{~min}^{-1}\end{array}$ & $\begin{array}{c}\mathrm{SA}_{\text {in }} \\
\mathrm{I}_{s} \min ^{-1}\end{array}$ & $\begin{array}{c}\mathrm{n}_{\text {bell }} \\
\min ^{-1}\end{array}$ & $\begin{array}{l}\mathrm{HV} \\
\mathrm{kV}\end{array}$ \\
\hline 300 & 300 & 240 & 50000 & 65 \\
\hline
\end{tabular}


ERBS. To determine the velocity components in three spatial directions, the flow field was scanned horizontally in two spatial directions at a fixed axial distance to the bell-edge. The scanned lines were measured selectively in an increment of $5 \mathrm{~mm}$. The measurement of a single position was stopped at the termination criterion of 10000 particles, and the mean value and the mean square error were calculated. The results are used to validate a turbulence model study (See "Simulation of the airflow field" section) focusing both close to the bell-cup $(\mathrm{d}=10 \mathrm{~mm})$ and close to the plate $(\mathrm{d}=180 \mathrm{~mm})($ Fig. 4$)$.

\section{Determination of particle size distribution}

The volumetric particle size distribution was measured using a SPRAYTEC RTS 5001 of the company Malvern Instruments. To minimize evaporation effects of the paint droplets on the result, the measurements were carried out at a $45^{\circ}$ angle and distance of $25 \mathrm{~mm}$ to the bell-edge. The optics of the device are protected by an attached tube against contamination by paint overspray. It is essential to keep the distance between bell-edge and measuring volume as small as possible to reduce evaporation effects, but it must be ensured that

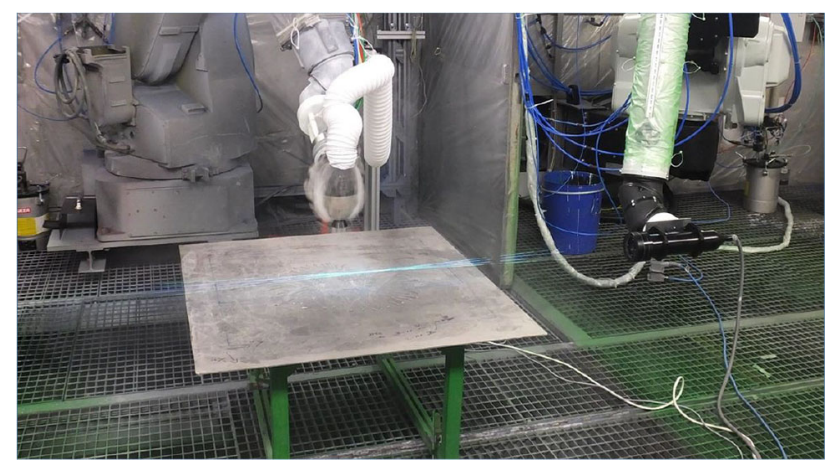

Fig. 4: Measurement setup to determine airflow velocities of shaping air by means of LDA

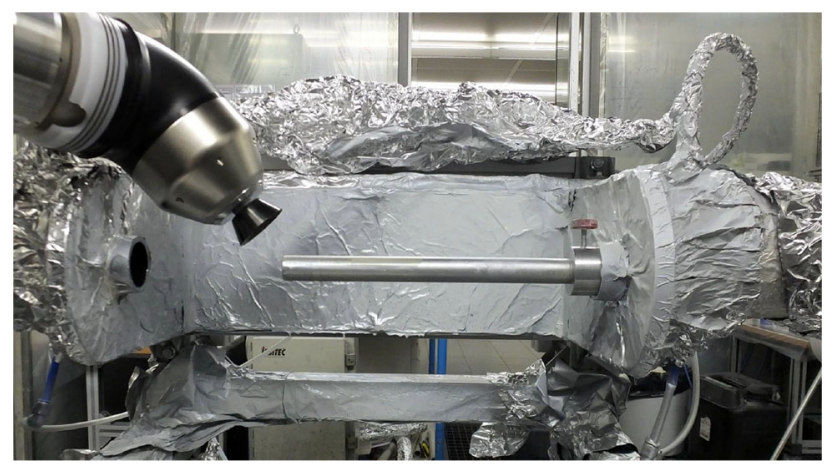

Fig. 5: Measurement setup to determine particle size distribution by means of laser diffraction the primary and secondary atomization is completed (Fig. 5).

\section{Determination of painting-specific data}

The paint application was executed by a painting robot moving the TCP with $180 \mathrm{~mm} \mathrm{~s}^{-1}$ at a distance of $200 \mathrm{~mm}$ parallel to the plate, which was placed horizontal in the center of a table (see Fig. 6). After the paint cured, the film thickness was measured by a magneto-inductive device perpendicular to the direction of painting, from which the so-called dynamic spray pattern could be obtained. At the same time, the discharge current at the background table was measured, from which the charge per time was calculated in the steady state.

\section{Numerical methods}

This section describes the scene, setup, and settings of the numerical simulations.

\section{Domain, grid and boundary settings}

The numerical simulations were carried out using the commercial computational fluid dynamics code ANSYSFluent. For this study, the ERBS was positioned in the center of a cylindric domain (radius $=1.1 \mathrm{~m}$, height $=0.8 \mathrm{~m}$ ) above the plate with a radius of $0.5 \mathrm{~m}$. The block-structured grid of the fluid domain contained about 17.36 million cells having a mean first prism layer height of $30 \mu \mathrm{m}$ around the bellcup and $150 \mu \mathrm{m}$ on the top of the plate. A velocity-inlet of $0.3 \mathrm{~ms}^{-1}$ was applied to the top boundary of the fluid domain to map the downdraft velocity of the paint booth air, whereby the sides and the bottom of the domain were set as pressure-outlets. The measured volumetric flow rates of the inner and outer shaping air were calculated into a mass flow rate using the International Standard Atmosphere ISO2533 and were

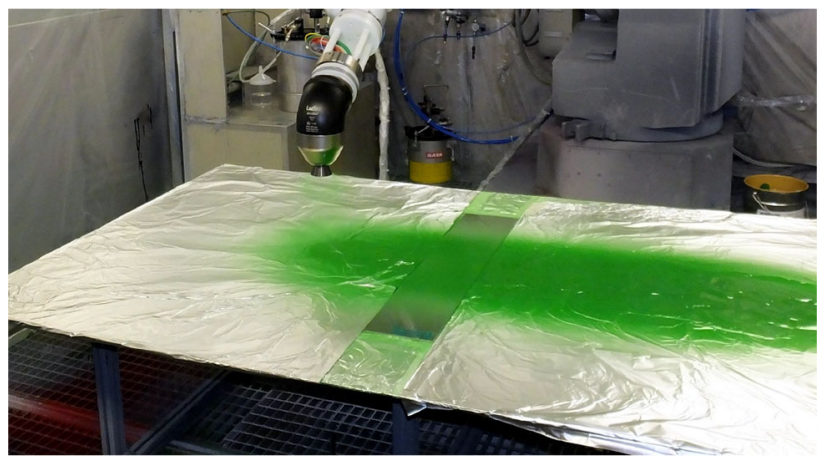

Fig. 6: Measurement setup to determine film thickness distribution-dynamic spray pattern 


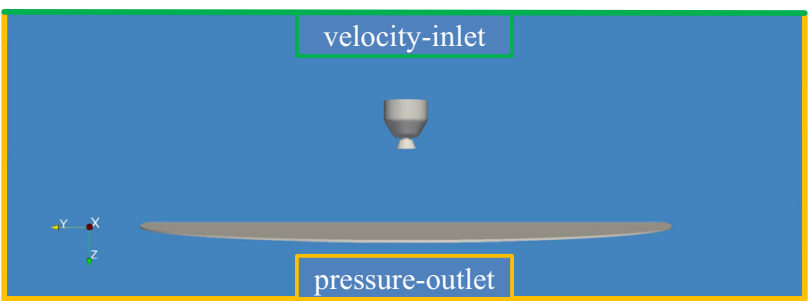

Fig. 7: Sketch of the fluid domain including the ERBS and the plate to be painted

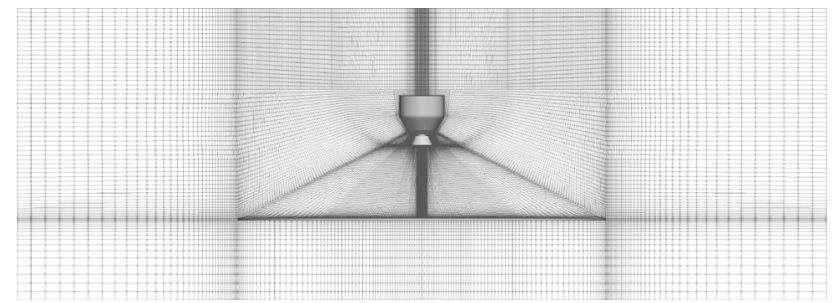

Fig. 8: Block-structured grid on a cross section through the center of the fluid domain

then applied as massflow-inlets on the top of shaping air nozzles. The rotation of the bell-cup was taken into account by setting the angular velocity on the walls of the bell-cup (Figs. 7 and 8).

\section{Turbulence model and solver settings}

In previous studies on ERBS, various 2-equation turbulence models, as well as Reynolds-Stress models, were used. In order to investigate the capability of the turbulence models for the strongly swirled airflow, a study using different modeling approaches was completed. The airflow results of the k- $\omega$ SST model are compared to the results of a Linear Pressure-Strain Reynolds Stress Model (RSM), and ANSYSFluents Improved Delayed Detached Eddy Simulation (IDDES) using the $\mathrm{k}-\omega$ SST as a sub-model. A second-order upwind was used as a discretization scheme, and the SIMPLEC algorithm with a fixed time step size of $\Delta \mathrm{t}=1 \times 10^{-4} \mathrm{~s}$ was chosen as the solver. With this time step size, a maximum CFL number of approximately 100 close to the shaping air nozzles was achieved on the generated grid. As the flow at the shaping air nozzles was almost linear, the error was not considered to be significant. However, the time step size could not be further reduced due to the high computational effort.

\section{Discrete phase model: motion of particles}

The motion of paint droplets were represented by inert and spherical particles computed through ANSYSFluents discrete-phase-model (DPM) in an Euler-La-

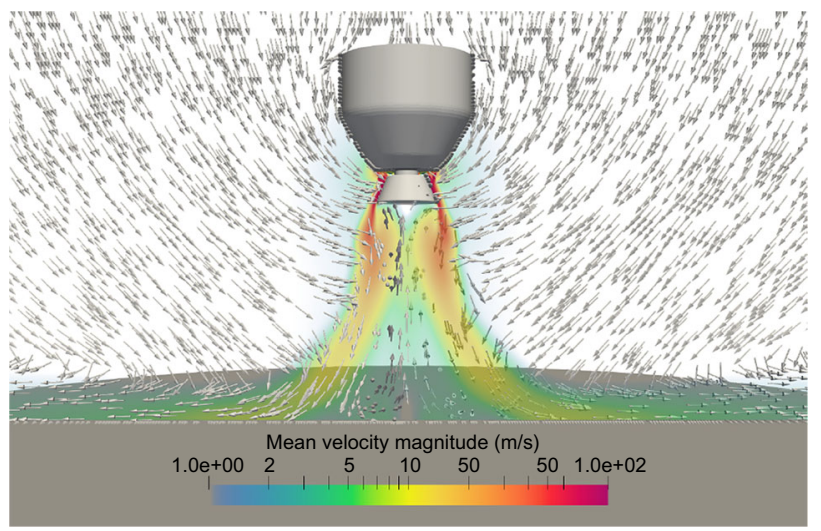

Fig. 9: Mean velocity magnitude of the shaping airflow field without droplets simulated using the k- $\omega$ SST turbulence model

grange framework. To capture the effects of the interaction of the particles with the flow and the electrostatic field, a two-way coupling was used and implemented, respectively. ${ }^{10,11,14} \mathrm{~A}$ random walking model was used to capture the turbulence effects on the trajectories of the particles. As a boundary condition, the particles were reflected on the bell-cup, trapped on the top of the plate and escaped on all other walls, inlets and outlets. A droplet deposition model was not used in this study because the effect on film thickness seemed very unlikely. The modeling of initial droplet conditions of the discrete phase are explained in detail in "Modeling initial droplet conditions" section.

\section{Simulation of the airflow field}

Since the airflow has a decisive influence on the resulting spray pattern, its accurate simulation is an essential basis for paint application simulations. The simulation must, therefore, be able to map the typical flow characteristics, such as vortex with backflow, distinct stagnation point on the object, and continuous air entrainment into the main flow field. Figure 9 shows the airflow field without particles, which was simulated using the k- $\omega$ SST turbulence model. The mean values were calculated over $0.5 \mathrm{~s}$ flow time. Qualitatively, the result shows all flow features of an ERBS. In particular, the backflow in the center of the flow field is characteristic of a strongly swirled shaping airflow field of ERBSs. ${ }^{15}$ However, it is well known that 2-equation turbulence models have limitations in predicting swirled impinging flows. In order to evaluate the accuracy and applicability, the results of the k- $\omega$ SST are quantitatively compared with the simulation results of the RSM and the IDDES as well as with experimental LDA data (see Fig. 10). Figure 10 shows the axial, tangential and radial velocity components at a distance of $\mathrm{d}=10 \mathrm{~mm}$ and $\mathrm{d}=180 \mathrm{~mm}$. In general, all turbulence models show an acceptable agreement with 

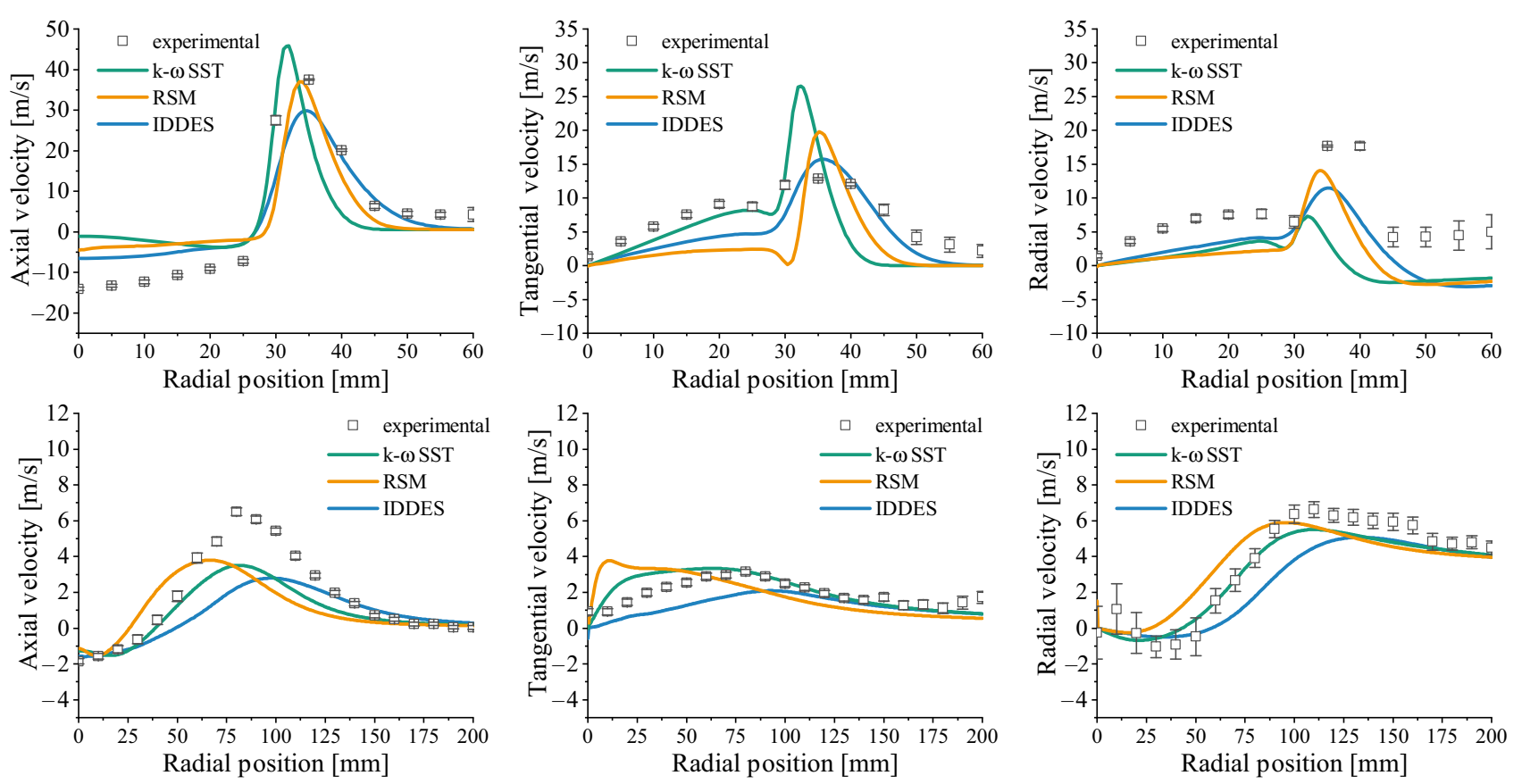

Fig. 10: Comparison of simulation results using three different turbulence models with experimental LDA data. The experimental graph shows the mean and $95 \%$ confidence. The velocity components axial (left column), tangential (mid column), and radial (right column) are compared at $d=10 \mathrm{~mm}$ (upper row) and $\mathrm{d}=180 \mathrm{~mm}$ (lower row)

the experimental data. However, each turbulence model has strengths and weaknesses in different flow regions and velocity components. Thus, the k- $\omega$ SST turbulence model shows significantly higher axial and tangential velocity than the IDDES near the bell-cup. Close to the plate, all simulation results show substantially lower axial velocity with good agreement in the radial velocity component. If the axial to radial velocity ratio in the experiment and the simulation are too far apart, the particles are inevitably transported outwards shortly before the plate, which widens the spray pattern. It can also be seen that the tangential velocity of RSM is overestimated in the center. As a result, the swirl strength of the flow increases and will be forced apart, and thus, higher radial velocities are generated, as it can be seen in the results near the plates.

\section{Modeling initial droplet conditions}

In this section, we propose an injection model for the initial droplet conditions-position, velocity, size, and charge-for an ERBS.

\section{Injection model: droplet position}

This ERBS has a notably formed bell-edge, which is often called a thick edge. Part of the paint climbs up this thick edge so that the atomization process takes place in a spread range of the axial position. This spread is visualized by a high-speed image close to the

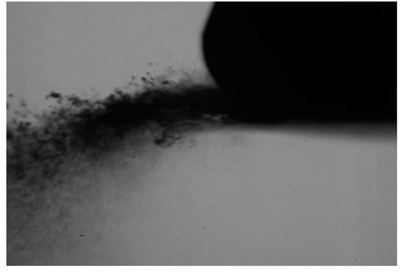

(a) Shadow image of disintegration process

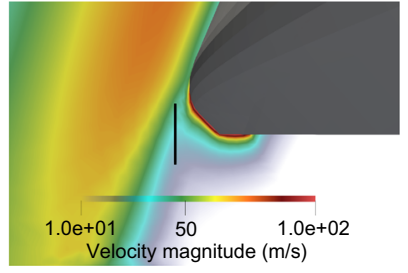

(b) Injection positions visualized by a black line; colorbar shows airflow velocity magnitude
Fig. 11: Visualization of the disintegration process at the bell-edge (a) and the injection position in the simulation (b)

bell-cup, see Fig. 11a. It is assumed that all fluid elements (droplets and/or ligaments) will pass the black plane shown in Fig. 11b, and this was also validated in the primary breakup simulation in the near bell region [see reference (9)]. The radial offset position $r_{\text {off }}$ is therefore defined at a fixed value of $r_{\text {off }}=0.75 \mathrm{~mm}$ from the bell-edge (end of flat part). In this case, 180 injection points that were uniformly distributed around the bell-cup were used. The entire particle size distribution is injected from each point per DPM iteration. To enhance the influence of the position on the result, the injection position was deliberately fixed for all particle sizes. Generally, the injection points should be distributed randomly in small areas to avoid overloading of the computational cells in the injection area and thus to achieve faster convergence. 


\section{Injection model: droplet velocity}

In paint application processes, the disintegration mode of the paint by ligament formation is preferred to direct droplet formation and sheet atomization, due to the resulting narrow, stable, and fine particle size distribution. Typically, a spiral formation of the ligaments at the bell-edge occurs visually (see Fig. 12 in green). The ligament formation begins at the bell-edge, where a fluid element with a velocity and direction of the vector $v_{0, r e s}$ from the bell-cup surface. In the following time steps (visualized by dots), the bell-cup rotates by an angle $\varphi$. The straight-line direction of the fluid element and the angular velocity of the bell-cup create a spiral-shaped ligament. The mathematical description of these ligaments, assuming that no forces act on a massless fluid element, is done by a simple geometric model. Due to the paint viscosity and the associated internal forces in the ligament, the direction of trajectory in the geometric model is bent. The angle $\alpha$ in the injection point thus increases with increasing internal forces and shifts the ratio of radial to tangential velocity components in the direction of the tangential velocity.

The initial vectors at the bell-edge are defined by the rotational speed and also by the film flow velocity on the bell. The tangential film flow velocity $v_{0, t a n}$ of the paint film is calculated assuming no-slip conditions on the surface of the bell-cup and homogeneous velocity distribution within the film. Reference (2) derived a formula for a simple cone to calculate the radial film flow velocity on the bell-edge. Assuming a Newtonian fluid and neglecting the circumferential and sinking speed, the radial velocity $v_{0, \text { rad }}$ will be obtained by

$v_{0, \text { rad }}=\left(\frac{9 \rho \omega^{2} \sin \beta Q^{2}}{32 \pi^{2} \eta_{s} r_{\text {bell }}}\right)^{1 / 3}$

where $Q$ denotes the quantity of paint delivery, $\omega$ the angular bell-cup velocity, $r_{\text {bell }}$ the radius of the bellcup, $\beta$ the cone angle at the bell-edge, $\rho$ the wet density of the paint, and $\eta_{s}$ the dynamic viscosity of the paint. Usually, paints are shear thinning and thixotropic liquids so that the viscosity is a function of the shear

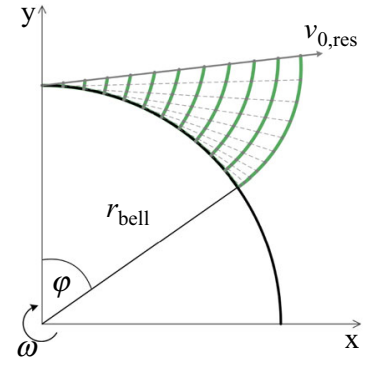

(a) Ligament formation

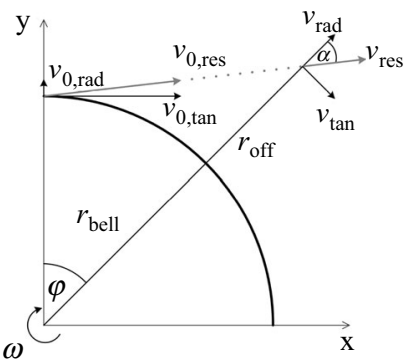

(b) Geometric model
Fig. 12: Sketch of the geometrical velocity model assuming ligament formation rate. Typically, automotive paint materials reach a viscosity plateau at approximately $20 \mathrm{mPa} s$ at very high shear rates ${ }^{16}$ measured through a capillary viscosimeter.

As a result of the energy loss during atomization, the ligament shape for calculating the acting forces, and the point of droplet formation, a slipfactor is introduced, which was applied to the vector $v_{0, r e s}$. The derivation of the slipfactor and angle $\alpha$ from analytical equations as well as material and process conditions is not trivial and is currently an unsolved problem. For this reason, the determination of the slipfactor and $\alpha$ was included in the optimization process.

\section{Injection model: droplet size distribution}

Although there is some numerical study of primary breakup in the near bell region, ${ }^{9}$ it is still quite difficult to simulate a fully developed droplet size distribution for the spray painting application, since very fine cell sizes have to be used. Instead of using the costly Volume-of-Fluid to DPM approach, a measured particle size distribution will be used. For this paint material, equipment, and process setting, the measured particle size distribution shows good agreement to the shape of a logarithmic normal distribution (see Fig. 13). The characteristic distribution values $d_{32}$ (Sauter mean diameter) and $d_{v}(50)$ (volumetric median) are in a typical range of automotive paint sprays.

\section{Injection: droplet charge}

Through the contact of paint on the inner bell-cup side, its surface is getting charged. After the atomization process and the resulting surface enlargement, the charge is distributed over the paint droplets, where currently two models are dominating in spray painting simulations. The first model assumes that the charge is distributed proportionally to $r^{2}$ of the droplet, whereas the second model assumes that the charge is distributed proportionally to $\sqrt{r^{3}}$. The latter is based on the equation of the Rayleigh-limit, where the maxi-

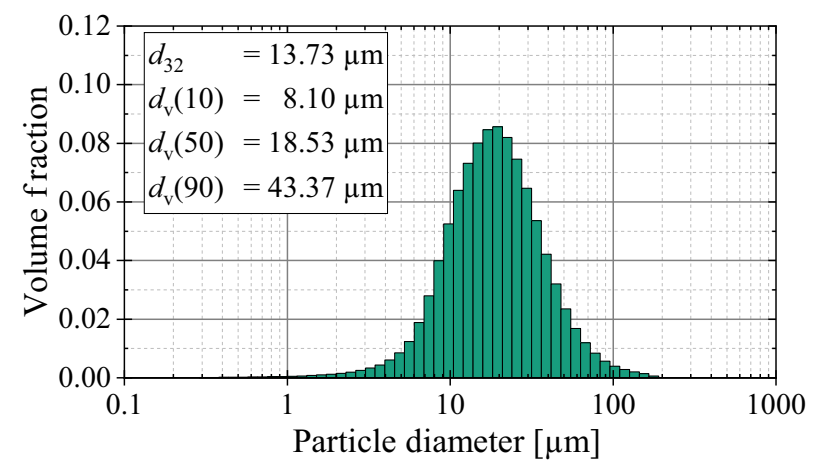

Fig. 13: Droplet size distribution 
mum charge on a droplet is calculated before it becomes unstable and disintegrates into smaller droplets.

$$
q_{\text {ray }}=8 \pi \sqrt{\varepsilon_{0} \gamma r^{3}}
$$

In this equation, $\varepsilon_{0}$ denotes the permittivity of the vacuum, $\gamma$ the surface tension of the paint and $r$ the droplet radius. The charge of the droplets in the injection model was calculated according to the equation of Rayleigh-limit. Since the surface charge is only a small percentage of the Rayleigh-limit, the Rayleigh-limit coefficient Coeff ray will be introduced and applied on the equation of Rayleigh-limit. The Rayleigh-limit coefficient is assumed in this model to be constant over all particle sizes. The dependence on the radius defines the total charge on the drop.

$$
q_{\text {sim }}=\text { Coeff }_{\text {ray }} q_{\text {ray }}
$$

From the experimentally measured discharge current, the Rayleigh-limit can be calculated at given particle size distribution assuming $100 \%$ paint transfer efficiency. In this way, the experimentally determined Coeff ray of $1.1 \%$ was calculated from the measured transferred charge per second of $11.37 \times 10^{-6} \mathrm{C} \mathrm{s}^{-1}$. Since the transfer efficiency in the experiment for ERBS using direct charging mechanism lies in the range of $80 \%$ to $95 \%$, it is meaningful to increase Coeff ray. To obtain a rough estimation of Coeff ray range, the experimentally calculated value $($ Coeff ray $=1.1 \%)$ should be increased by $20 \%$ to $40 \%$ so that in this study the range between Coeff ray $=$ $1.32 \%$ and Coeff ray $=1.54 \%$ is assumed to be the target range. Due to the uncertainty in the overspray estimation and the amount of charge it contains, the Coeff $f_{\text {ray }}$ is included in the optimization process.

\section{Metamodel-based optimization of injection model coefficients}

In this step of the framework, a metamodel-based optimization strategy will be used to efficiently determine the injection model coefficients.

\section{Design of experiments: sampling points}

The framework starts with the definition of a feasible space of the injection model parameters, whereby this should cover a wide range of the parameters. In this design space, the axial injection position was based on the area of atomization mentioned in "Injection model: droplet position" section. The axial velocity is also defined for negative values since the thick belledge may lead to an upward flow of the paint film. The maximum value for the axial velocity is defined as
$30 \mathrm{~ms}^{-1}$, which represents an overestimation due to the small airflow velocity in that area (see Fig. 11b). Since the internal forces in the ligament and the energy loss during atomization are not known, the slipfactor and the angle $\alpha$ are investigated over an extensive range. Table 2 shows the design space by minimum and maximum values of the parameters as well as constant values. Typically, Latin-Hypercube sampling methods are used for computer experiments to train regression models. Particularly for practical applications, the »maximin « method is often used, which maximizes the minimum distance between sampling points and therefore avoids too narrow points. This method was also chosen to create 150 sample points for this study.

\section{Conduction and evaluation of simulations}

The simulations were performed on the cluster of the High Performance Computing Center Stuttgart (HLRS). To reduce the total duration time of the simulations, a task-based parallelization is strongly recommended. In this series, ten simulations were performed simultaneously. In each simulation, a presimulated mean airflow field from the turbulence study is loaded at the beginning. Particles are then injected over $0.75 \mathrm{~s}$, whereby the first $0.25 \mathrm{~s}$ are not taken into account in the evaluation, as the spray has not reached a steady state. With very fine computational grids on the objects to be painted, the so-called binning effects often occur in the calculation of the film thickness. This is caused by large particle volume deposits in a very small computing cell. For painting simulations, it is recommended to decouple the computational cells of the airflow from cells of the film thickness. Therefore, particles deposited on the plate are sampled calculated into the film thickness in a postprocessing step. The dynamic spray pattern can be calculated on the one hand directly from the point cloud of the sampled data via a kernel density estimation (KDE) or on the other hand via an initially calculated static spray pattern (see Fig. 14) using a new generated structured.

In the simplest case, the conversion from static to dynamic spray pattern can be done by integrating the film thickness along a coordinate axis. Since binning effects create noise within the calculated spray pattern,

\begin{tabular}{|c|c|c|c|c|}
\hline Parameter & Description & Min & Max & Unit \\
\hline Axial position & AX_POS & -0.5 & 0.5 & $\mathrm{~mm}$ \\
\hline Axial velocity & AX_VEL & -5 & 30 & $\mathrm{~ms}^{-1}$ \\
\hline Slipfactor & SLIPFACTOR & 25 & 95 & $\%$ \\
\hline Angle $\alpha$ & ANGLE & 76.62 & 85 & $\circ$ \\
\hline Coeff $_{\text {ray }}$ & COEFF_RAY & 0.5 & 1.5 & $\%$ \\
\hline Constants & & Value & & Unit \\
\hline Radial Position & & 0.75 & & $\mathrm{~mm}$ \\
\hline
\end{tabular}

Table 2: Feasible space of injection model parameters 


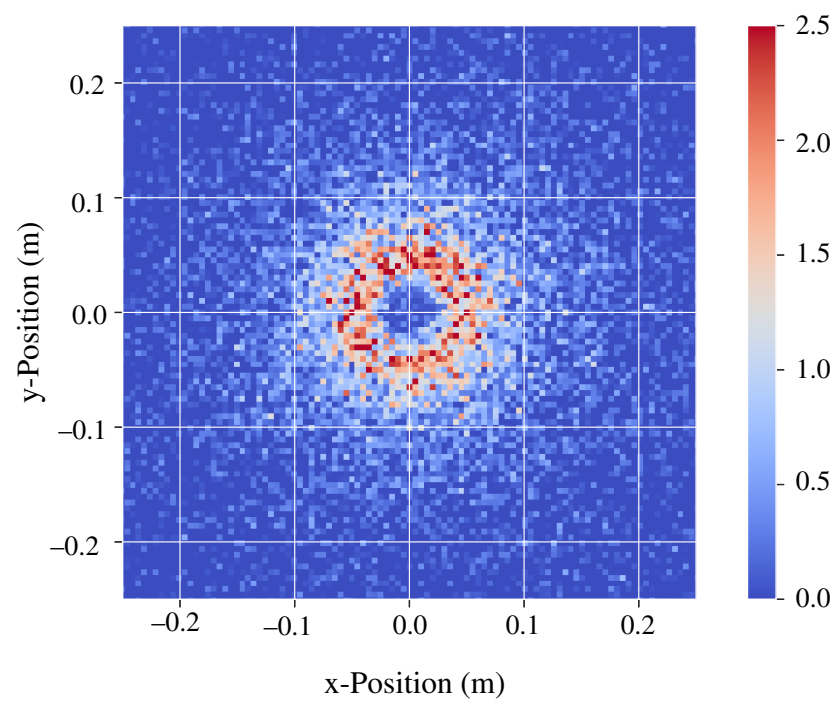

Fig. 14: Static spray pattern at a resolution of $5 \mathrm{~mm} \times 5 \mathrm{~mm}$; the colorbar represents the film thickness in $\mu \mathrm{m}$

it is recommended to average the results by rotating the axis around the center of the static spray pattern. An inverse Radon transformation can efficiently calculate this rotatory averaging over $360^{\circ}$ around the pattern center, which is why this method is preferred to the costly KDE. Figure 15 shows the result of the three methods discussed for calculating a dynamic spray pattern from a static (nonmoving atomizer) simulation.

It can be seen that the inverse Radon transformation is almost identical to the result of the integral method, but due to the $360^{\circ}$ rotation, the curve is much smoother and more symmetrical. Because of the rotation, this method is suitable only for static and horizontal simulation scenes using a flat plate. The KDE also generates a continuous spray pattern curve, but the maximum differs significantly from the other two methods. This deviation can be attributed to the known problem of choosing the kernel and the bandwidth of the estimator. Nevertheless, KDE methods are helpful tools to calculate the film thickness on complex 3D parts, as it is shown in reference (17).

In addition to the important spray pattern, further painting-specific parameters are calculated and evaluated from the simulations and partly included in the optimization. These values are briefly explained in the following enumeration:

- RESIDUAL: Sum of least squares between simulated and experimental film thickness distribution

- PW50: Pattern width 50, the full width at half maximum of the film thickness distribution

- SUM_CHARGE: Sum of the charge from the droplets deposited on the plate

- TE_WEIGHT: Mass-based transfer efficiency, ratio between deposited to injected mass of paint

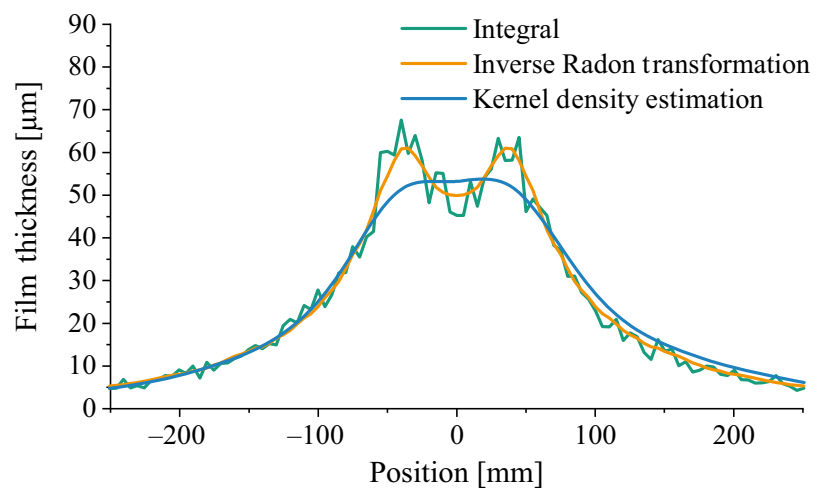

Fig. 15: Comparison of methods to calculate dynamic spray pattern

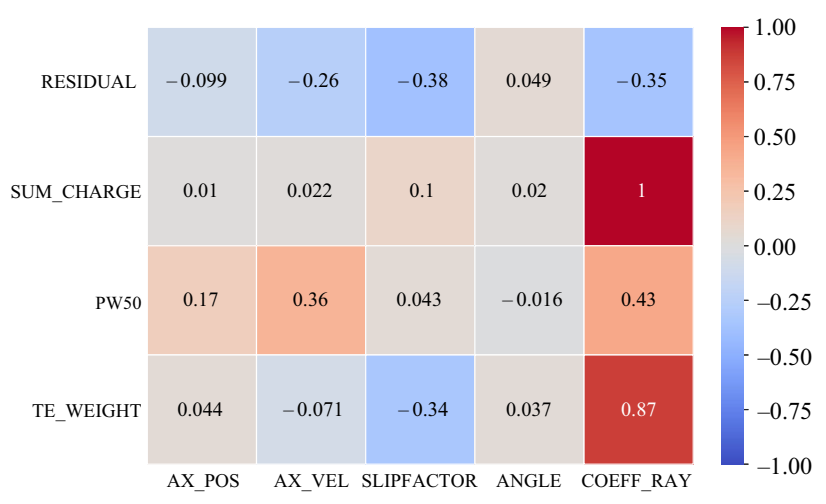

Fig. 16: Correlation matrix using Spearman's correlation coefficient

\section{Principle component analysis}

From the results of the statistical design of experiments, the correlations of the input parameters to the objective values were calculated using a Spearman's correlation, which is a nonparametric measure of rankorder correlation. The measure describes the strength and direction of a monotonic association between two variables. Values close to -1 (perfect negative correlation) and 1 (perfect positive correlation) represent a strong monotonic correlation, whereas values around 0 represent a very weak monotonic relationship between two variables.

Figure 16 shows that the correlation between axial position and painting specific values is very weak and, therefore, does not represent a principal component. The same applies to the angle $\alpha$. The correlation between the COEFF_RAY and the transferred charge and the transfer efficiency is particularly high as well as highly linear. The well-known expansion of the spray with increasing charge is also found in the correlation coefficient between COEFF_RAY and the PW50 with a moderate value of 0.43 . In the following optimization steps, only the principal model parameters AX_VEL, SLIPFACTOR, and COEFF_RAY are considered. 


\section{Training of metamodels}

Global multiobjective optimizations often require many function evaluations to calculate a Pareto front. Since each function evaluation means a painting simulation, two metamodels were created first. Metamodels or surrogate models are compact, mathematical approximations that map the multivariate input/output behavior of complex systems. In this study, a metamodel was created for two objectives-RESIDUAL and residual of transferred charge (RESIDUAL CHARGE), which is the quadratic error between simulated and measured value. For the RESIDUAL of the spray pattern, a three-parameter Kriging model from the »SMT-Surrogate Modeling Toolbox« Python toolbox ${ }^{18}$ was used.

In common machine learning practice, the quality of the prediction for regression models is determined by the coefficient of determination $R^{2}$. Because of the few available sample points, it is recommended to determine a mean $R^{2}$ via the »leave-one-out cross-validation « method. In this method, the metamodel was trained with $\mathrm{n}-1$ sample points, and $R^{2}$ is evaluated at the omitted location. Afterward, a mean $R^{2}$ value was computed from all sample point evaluations. The learning curve of the metamodel can thus be monitored for the current number of sample points, see Fig. 17. This three-parameter metamodel learns particularly active within the first 60 sample points. At 100 sample points, the metamodel reaches a constant level above a $R^{2}>0.9$, which allows acceptable predictions to be made for this application. To accelerate performance and to reduce computational effort, the learning process should be terminated after reaching $R^{2}>0.9$ for ten consecutive training sets.

Figure 18 visualizes the influence of the injection model parameters on the RESIDUAL of the spray pattern. The visualization was done by evaluating the Kriging predictions on a uniform grid in each dimension.

The evaluation of the parameter impacting on the spray pattern result shows a clear trend toward high slipfactor, a Rayleigh-limit coefficient of approxi-

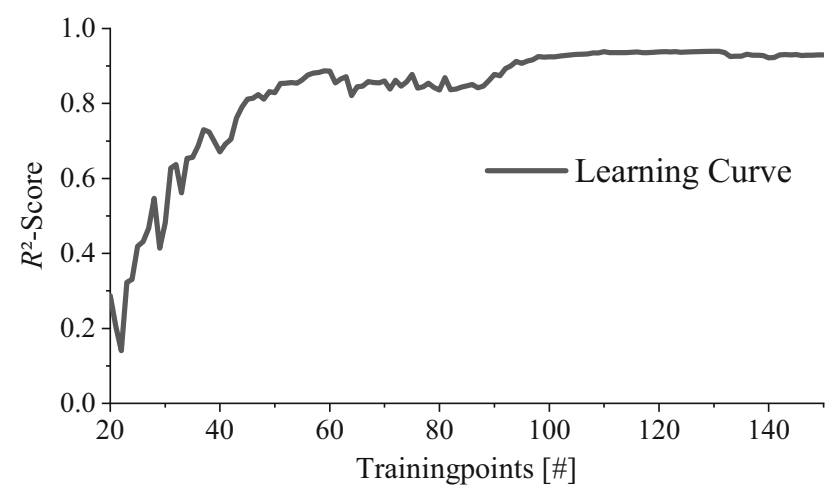

Fig. 17: Learning curve of the metamodel scored by $R^{2}$ mately $1.4 \%$, and a weak trend toward low axial velocities. These results also follow the expected theoretical properties of injection in the velocity reduced zone of the thick bell-edge.

A simple 1D interpolation is a sufficient metamodel for the second objective function because only the charge on the droplet acts as a principal component. The transferred charge on the plate scales linearly with the charge on the droplets, so the residual is also a quadratic function. The residual between simulated and experimental values reaches a minimum at a Rayleigh limit of $1.39 \%$ (Fig. 19).
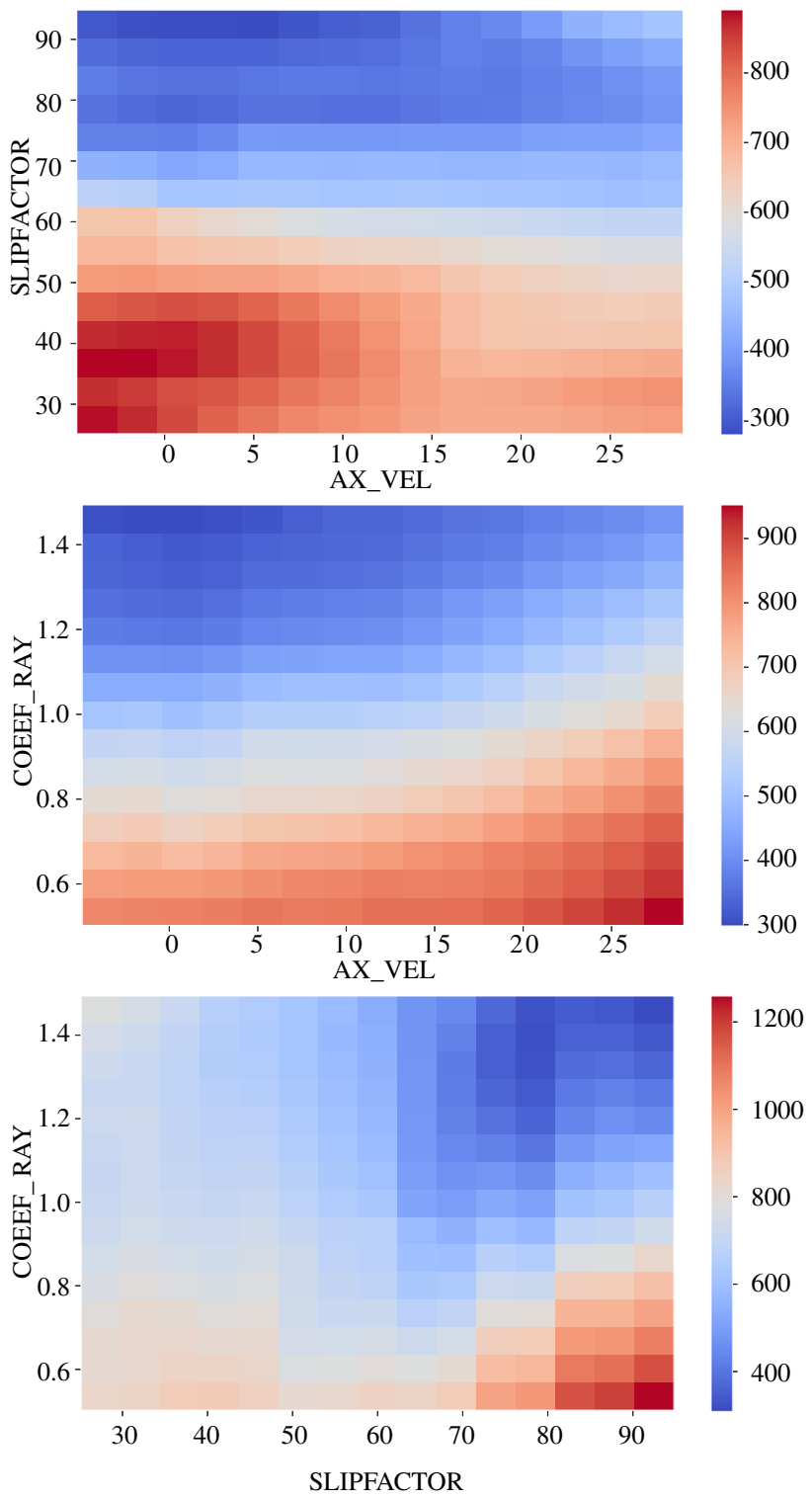

Fig. 18: Pairplots of input parameters of the Kriging model trained with the simulation data from the RSM turbulence model. The colorbar visualizes the values of the RESIDUAL 


\section{Multiobjective optimization}

The Pareto optimization of the principal components in the injection model is based on the minimization of two objective functions and previously trained metamodels. The first objective function $\mathrm{f} 1$ is to minimize the RESIDUAL, and the second objective function $\mathrm{f} 2$ is to minimize the least square between measured and simulated charge per time (discharge current) on the plate. It is possible to define further objective functions, e.g., based on LDA velocity data, which could lead to more precise solutions. However, for an efficient application, it is advisable to limit the objective functions to existing or easily generated characteristics of the painting process.

The multiobjective optimization was performed using the Nondominated Sorting Genetic Algorithm III (NSGA-III) from the Python toolbox $»$ pymoo «. ${ }^{19}$ Details on the implementation of the NSGA-III algorithm into the "pymoo « toolbox can be found in reference (20). The various parameters of the NSGAIII algorithm are listed in Table 3 with the descriptions specified in the toolbox.

Minimizing the two objective functions $\mathrm{f} 1$ and $\mathrm{f} 2$ leads to a Pareto front and a single point of Paretooptimal solution. Due to the different scaling for $\mathrm{f} 1$ and $\mathrm{f} 2$, it is recommended to use a pseudo-weighted ${ }^{23}$ decision to determine the Pareto-optimal point. In this

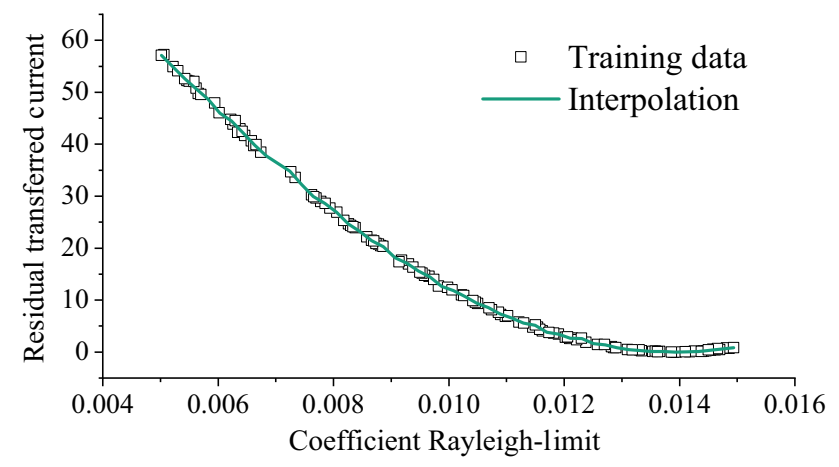

Fig. 19: 1D metamodel for the residual of transferred charge to the plate built using a quadratic interpolation method study, the optimal initial droplet conditions were computed for both the RSM and the k- $\omega$ SST turbulence model (see Table 4).

The optimized initial droplet conditions were validated by one additional simulation to evaluate the error. In both cases, the deviation of the simulated to the experimental charge per time is less than $2 \%$. The simulated spray pattern also shows an acceptable fit to the experimentally determined result (see Fig. 20).

In particular, the axial velocity for RSM and $\mathrm{k}-\omega$ SST differs significantly. These discrepancies relate to the results of the turbulence study, where in both turbulence models the axial velocity near the plate was clearly underestimated. However, the k- $\omega$ SST simulations have a good agreement in the contour of the curve and the position of the peaks, as well as in the tangential and radial velocity. Consequently, the optimization process tries to compensate for the error of the underestimated axial velocity. For the RSM, the underestimated axial velocity applies as well, but the entire shaping airflow field is slightly narrower than the $\mathrm{k}-\omega$ SST simulations so that a too narrow shaping airflow field compensates the missing axial impulse. Furthermore, the high initial axial velocity in the k- $\omega$ SST case leads to a lower Rayleigh-limit because the high initial impulse forces larger particles to be deposited on the plate. For this particular ERBS with a thick bell-edge, it is unlikely that such a high axial velocity is present for the initial drop conditions and is, therefore, an indicator for incorrect air flow prediction. However, the optimization process itself can efficiently

Table 4: Optimized injection model coefficients for RSM and $\mathbf{k}-\omega$ SST turbulence model cases using the metamodel-based optimization framework

\begin{tabular}{llll} 
Parameter & \multicolumn{1}{c}{ RSM } & \multicolumn{1}{c}{ k- $\omega$ SST } & Unit \\
\hline AX_POS & 0 (fixed) & 0 (fixed) & $\mathrm{mm}$ \\
AX_VEL & 3.5 & 18.6 & $\mathrm{~ms}^{-1}$ \\
SLIPFACTOR & 88.0 & 86.8 & $\%$ \\
ANGLE & 76.62 (fixed) & 76.62 (fixed) & $\circ$ \\
COEFF_RAY & 1.45 & 1.31 & $\%$ \\
\hline
\end{tabular}

\section{Table 3: Parameter of NSGA-III}

Parameter

Population size

Sampling method

Reference points

Crossover operation

Mutation operation

Stopping criteria

Decision making

Method

Value

Latin-Hypercube
Das-Dennis $^{21}$
Point crossover $^{22}$
Polynomial mutation $^{22}$
Maximum generations $^{\text {Pseudo-weights }}{ }^{23}$

Latin-Hypercube

Das-Dennis ${ }^{21}$

Point crossover ${ }^{22}$

Maximum geners

Pseudo-weights ${ }^{23}$

100
-
n_partitions $=25$
n_points $=2$
eta $=30$, prob $=0.9$
1000
$f 1=0.5, f 2=0.5$




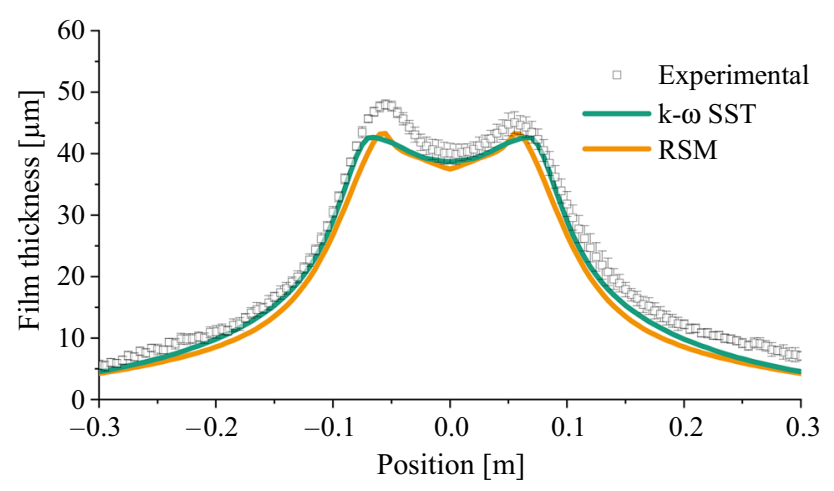

Fig. 20: Comparison of experimental and simulated spray pattern using the Pareto-optimal initial droplet conditions

determine the Pareto-optimum. At this point, the physically meaningful range of initial droplet conditions for different ERBS types is not established. It must be defined by further experimental investigations near the bell-edge as well as by the support of additional sub-models in the injection modeling.

\section{Discussion}

The framework presented here to determine the initial droplet conditions for ERBS simulations has been realized using a simple injection model, experimental data, and a metamodel-based optimization strategy. Within the framework, uncertainties occur both in the determination of the experimental data and in the paint application simulation.

A statistically relevant mean value should be formed to reduce the experimental uncertainties. Experience has shown that variations of up to $15 \%$ can occur, especially for spray pattern and measurement of discharge current on the plate. However, for economic and plant availability reasons, a sufficient number of experiments cannot always be guaranteed, so that there is a risk of calibrating to a spray pattern at the boundary of the variation.

The particle size determination could alternatively be measured using a Phase-Doppler Anemometer (PDA) by systematically evaluating the spray point by point and converting the obtained number distribution into a volume distribution under consideration of droplet velocities. The disadvantage of the PDA method is that the measurements with opaque paint materials do not work, and are, respectively, very error-prone. Besides, under economical aspects for industrial applications, the costs for PDA measurements are many times more expensive. Therefore, measuring methods with laser diffraction are recommended to measure the particle size distribution as an input condition for painting simulations.

The proposed injection model is a simple first-order approximation of the highly complex processes during primary atomization at the bell-edge. With the help of the principal component analysis and metamodels, it is possible to adapt further and optimize the injection models. In particular, the initial velocity independent from particle sizes is currently a significant weak point. It is known that the particle relaxation times near the bell for particle sizes larger than $100 \mu \mathrm{m}$ are so high that they are not distracted much and retain their initial direction. Particle sizes smaller than $5 \mu \mathrm{m}$, on the other hand, are almost strongly coupled to the flow. The range of these initial velocities as a function of particle size is to be included in further investigations in the form of a crossflow submodel. In order to determine the range of velocities, complex experiments with optical measuring methods must be carried out in order to investigate the correlation between particle size and particle velocity. Furthermore, the results of the primary break-up volume-of-fluid simulations at the bell-edge of references (8) and (9) will be investigated and used to enhance and validate the injection models for ERBS.

The most significant impact on the simulated result is the correct prediction of the airflow. Modern ERBS tend to utilize swirled shaping air to increase its range of application. Particularly in the simulation with linear eddy-viscosity models, overproduction of turbulence at impinging flows is known, despite using a production limiter. In all turbulence models, the axial velocity was significantly underestimated. For simulations in an industrial environment with commercial simulation software, turbulence models such as the k- $\omega$ SST will continue to be used in the future. Since other preimplemented models also fail, further investigations and adjustments of the models have to be carried out in order to achieve high accuracy of the film thickness prediction.

It must be assumed that further changes in the model constants, e.g., turbulence or particle models, will also lead to changes in the initial particle conditions. In particular, the uncertainty of solvent evaporation from the droplets, which was not taken into account in the modeling, exists in this work. Particularly for solvent-based paints with low solids content, a drastic influence could be caused. The implementation of evaporation models is very complicated in the field of paint materials because interactions of complex solvent mixtures have to be modeled, which creates further hurdles in the generation of input data. However, water-based solvents could be considered using existing evaporation laws. A significant influence of droplet impingement models as well as droplet collision models film thickness distribution is currently estimated to be very low and is therefore not considered in these painting simulations.

The metamodel-based optimization is an efficient method to perform a multicriteria black-box optimization with moderate computational effort. The black box contains both the experimental input data and the painting simulations themselves. The previously discussed errors are wholly included. If the errors, such as the airflow field, are substantial, the optimizer will find 
solutions in the boundary area of the defined design space. Currently, this space is not completely described and limited by experiments, models, and assumptions, which will be investigated in further research and development.

From a holistic point of view, good agreement with the objectives could be achieved through this framework while painting complex parts, the alignment of the atomizer to the part surface changes, and as a result, the flow field is changed. Thus, the question of the validity range of the optimized parameters remains open. Further investigations with different distances and angles of the atomizer to the surface should provide clarity. However, the requirements for the correctness of the simulated airflow are also applied here.

\section{Conclusions}

In this paper, we have proposed a framework to systematically address the practical problem of initial drop conditions at ERBS for painting simulations. The framework is a combination of experimental input data, an injection model, and a metamodel-based optimization. First, the determination of the experimental data was discussed and its utilization in the framework graphically outlined. Second, the numerical cases and models were explained. A turbulence study with three turbulence models was performed and compared with experimental LDA data. A strong underestimation of the axial velocity component in all three turbulence models was clearly shown. The tangential and radial velocity, on the other hand, were simulated with reasonable accuracy. Furthermore, an injection model used to determine the initial drop conditions-position, particle velocity, particle size, particle charge-was introduced. A metamodel-based optimization then adjusted the model parameters. The results of the optimization show a good agreement with the defined painting-specific objectives. However, the optimization revealed weak points of the underestimated axial air velocity by setting high initial axial velocities for the droplets to compensate for the missing impulse. Further investigations will focus on the particle size-dependent initial velocity in the injection model and supporting the model development and validation by experimental investigations.

Acknowledgments Open Access funding provided by Projekt DEAL. This research was supported within the project »SelfPaint « by Fraunhofer-Gesellschaft zur Foerderung der angewandten Forschung e.V. The authors acknowledge for the support of the High Performance Computing Center Stuttgart HLRS.

Open Access This article is licensed under a Creative Commons Attribution 4.0 International License, which permits use, sharing, adaptation, distribution and reproduction in any medium or format, as long as you give appropriate credit to the original author(s) and the source, provide a link to the Creative Commons licence, and indicate if changes were made. The images or other third party material in this article are included in the article's Creative Commons licence, unless indicated otherwise in a credit line to the material. If material is not included in the article's Creative Commons licence and your intended use is not permitted by statutory regulation or exceeds the permitted use, you will need to obtain permission directly from the copyright holder. To view a copy of this licence, visit http://creativecommons.org/licenses/by/4.0/.

\section{References}

1. Bruin, S, "Velocity Distribution in a Liquid Film Flowing over a Rotating Conical Surface." Chemical Engineering Science, 24 (11) 1647-1654 (1969). https://doi.org/10.1016/00 09-2509(69)87029-6

2. Hinze, JO, Milborn, H, "Atomization of Liquids by Means of a Rotating Cup." Journal of Applied Mechanics, 145-153 (1950)

3. Makarytchev, SV, Xue, E, Langrish, T, Prince, R, “On Modelling Fluid Flow Over a Rotating Conical Surface." Chemical Engineering Science, 52 (6) 1055-1057 (1997). https://doi.org/10.1016/S0009-2509(96)00473-3

4. Mehrhardt, E, Brauer, H, "Zerstäubung von flüssigkeiten mit rotierenden scheiben." Forschung im Ingenieurwesen, 46 (1) 26-30 (1980). https://doi.org/10.1007/BF02560879

5. Glahn, A, Busam, S, Blair, MF, Allard, KL, Wittig, S, "Droplet Generation by Disintegration of Oil Films at the Rim of a Rotating Disk." Journal of Engineering for Gas Turbines and Power, 124 (1) 117 (2002). https://doi.org/10. 1115/1.1400753

6. Liu, J, Yu, Q, Guo, Q, "Experimental Investigation of Liquid Disintegration by Rotary Cups." Chemical Engineering Science, 73 44-50 (2012). https://doi.org/10.1016/j.ces.20 12.01.010

7. Ahmed, M, Youssef, MS, "Influence of Spinning Cup and Disk Atomizer Configurations on Droplet Size and Velocity Characteristics." Chemical Engineering Science, 107 149-157 (2014). https://doi.org/10.1016/j.ces.2013.12.004

8. Shen, B, Ye, Q, Tiedje, O, Domnick, J, "Primary Breakup of Liquids using a High-Speed Rotary Bell Atomizer for Spray Painting Processes." In: Proceedings ILASS-Europe 2017. 28th Conference on Liquid Atomization and Spray Systems, Universitat Politècnica València, Valencia (2017). https://d oi.org/10.4995/ILASS2017.2017.4611

9. Shen, B, Ye, Q, Guettler, N, Tiedje, O, Domnick, J, "Primary Breakup of a Non-Newtonian Liquid Using a High-Speed Rotary Bell Atomizer for Spray-Painting Processes." Journal of Coatings Technology and Research, 16 (6) 1581-1596 (2019). https://doi.org/10.1007/s11998-019-002 31-4

10. Domnick, J, Scheibe, A, Ye, Q, "The Simulation of the Electrostatic Spray Painting Process with High-Speed Rotary Bell Atomizers. Part I: Direct Charging." Particle \& Particle Systems Characterization, 22 (2) 141-150 (2005). https://doi. org/10.1002/ppsc. 200400968 
11. Osman, H, Adamiak, K, Castle, GSP, Fan, HT, Simmer, J, "Comparison Between the Numerical and Experimental Deposition Patterns for an Electrostatic Rotary Bell Sprayer." In: ASME 2015 International Mechanical Engineering Congress and Exposition, American Society of Mechanical Engineers, New York, N.Y., p V02BT02A003 (2016). https://doi.org/10.1115/IMECE2015-50211

12. Colbert, SA, Numerical Simulations of Droplet Trajectories from an Electrostatic Rotary-Bell Atomizer (2006)

13. Toljic, N, Adamiak, K, Castle, G, Kuo, HH, Fan, HT, "Three-Dimensional Numerical Studies on the Effect of the Particle Charge to Mass Ratio Distribution in the Electrostatic Coating Process." Journal of Electrostatics, 69 (3) 189194 (2011). https://doi.org/10.1016/j.elstat.2011.03.008

14. Ye, Q, Domnick, J, Scheibe, A, Pulli, K, "Numerical Simulation of Electrostatic Spray-Painting Processes in the Automotive Industry." In: High Performance Computing in Science and Engineering' 04: Transactions of the High Performance Computing Center Stuttgart (HLRS) 2004, Springer Berlin Heidelberg, Berlin, Heidelberg, pp 261-275 (2005)

15. Guettler, N, Paustian, S, Ye, Q, Tiedje, O, "Numerical and Experimental Investigations on Rotary Bell Atomizers with Predominant Air Flow Rates." In: Proceedings ILASS Europe 2017. 28th Conference on Liquid Atomization and Spray Systems, Universitat Politècnica València, Valencia (2017). https://doi.org/10.4995/ILASS2017.2017.4650

16. Oswald, W, Goedecke, L, Ehrhard, P, Willenbacher, N, "Effect of Elongational Flow Behavior on Ligament Disintegration and Drop Formation by Mean of a High-Speed Rotary Bell Atomizer." In: ICLASS 2018, 14th Triennial International Conference on Liquid Atomization and Spray Systems, Chicago (2018)

17. Tafuri, S, Ekstedt, F, Carlson, JS, Mark, A, Edelvik, F, "Improved Spray Paint Thickness Calculation from Simulated Droplets Using Density Estimation." In: Proceedings of the ASME International Design Engineering Technical
Conferences and Computers and Information in Engineering Conference-2012, American Society of Mechanical Engineers, New York, N.Y., pp 339-347 (2012). https://doi.org/10. 1115/DETC2012-70821

18. Bouhlel, MA, Hwang, JT, Bartoli, N, Lafage, R, Morlier, J, Martins, JRRA, "A Python Surrogate Modeling Framework with Derivatives." Advances in Engineering Software, 102662 (2019). https://doi.org/10.1016/j.advengsoft.201 9.03.005

19. Blank, J, Deb, K, pymoo - Multi-objective Optimization in Python (2019). https://pymoo.org

20. Deb, K, Sundar, J, "Reference Point Based Multi-objective Optimization Using Evolutionary Algorithms." In: Cattolico, M (ed.) Proceedings of the 8th Annual Conference on Genetic and Evolutionary Computation - GECCO '06, p 635, ACM Press, New York, New York, USA (2006). https://doi.org/10. 1145/1143997.1144112

21. Das, I, Dennis, JE, "Normal-Boundary Intersection: A New Method for Generating the Pareto Surface in Nonlinear Multicriteria Optimization Problems." SIAM Journal on Optimization, 8 (3) 631-657 (1998). https://doi.org/10.1137/S 1052623496307510

22. Deb, K, Sindhya, K, Okabe, T, "Self-Adaptive Simulated Binary Crossover for Real-Parameter Optimization." In: Lipson, $\mathrm{H}$ (ed.) Proceedings of the 9th Annual Conference on Genetic and Evolutionary Computation-GECCO '07, p 1187, ACM Press, New York, New York, USA (2007). https://doi.org/10.1145/1276958.1277190

23. Deb, K, Kalyanmoy, D, Multi-Objective Optimization Using Evolutionary Algorithms. John Wiley \& Sons, Inc., New York, NY, USA (2001)

Publisher's Note Springer Nature remains neutral with regard to jurisdictional claims in published maps and institutional affiliations. 